\title{
Tacrolimus in Renal Transplantation
}

R. Shapiro, M.L. Jordan, V.P. Scantlebury, C. Vivas, H.A. Gritsch, J. McCauley, D. Ellis, N. Gilboa, S. Lombardozzi-Lane, P. Randhawa, A.J. Demetris, W. Irish, T.R. Hakala, R.L. Simmons, J.J. Fung, and T.E. Starzl

CACROLIMUS (Prograf-FK506) has been used since 1989 as a primary immunosuppressive agent in adult and pediatric renal transplant recipients at our institution. ${ }^{1.2}$ This report will summarize our experience with it over the past six years and will demonstrate its overall efficacy and superiority.

\section{Adults}

The early studies with tacrolimus, from 1989 through 1991, included a pilot series, a small randomized trial, and a larger nonrandomized group. ${ }^{1.3,4}$ When the outcome of these patients was compared with that in a roughly concurrent (although somewhat less challenging) group of cyclosporine (CyA)-treated patients, equivalent patient and graft survival rates were seen. However, improved secondary outcomes were noted in the tacrolimus-treated patients, including lower steroid and antihypertensive medication requirements, and lower serum cholesterol levels. ${ }^{4}$ This led to a second prospective, randomized trial, between August, 1991 and December, 1993, comparing two tacrolimus-based regimens, with and without azathioprine..$^{5-8}$ Earlier analyses of this trial (by intention to treat, without patient censoring) demonstrated excellent outcomes with tacrolimus overall, but with unclear benefit for azathioprine. The most recent analysis, with a mean follow-up of $33 \pm 10$ months, showed a one- and three-year actuarial patient survival of $95 \%$ and $92 \%$, with no significant difference between the two groups (Table 1). Overall one- and threeyear actuarial graft survival was $89 \%$ and $80 \%$, with worse results in the tacrolimus/azathioprine/prednisone group than in the tacrolimus/prednisone group. Overall one- and three-year actuarial graft survival in first cadaver recipients was $91 \%$ and $82 \%$, without a statistically significant differ-

Table 1. Tacrolimus in Renal Transplantation Adults $\mathbf{n}=\mathbf{3 9 7}$

\begin{tabular}{lccc}
\hline & Tacro/Pred & Tacro/Aza/Pred & Overall \\
\hline $\begin{array}{l}\text { Actuarial Patient Survival } \\
\quad 1 \text { year }\end{array}$ & $97 \%$ & $94 \%$ & $95 \%$ \\
$\quad 3$ year & $94 \%$ & $90 \%$ & $92 \%$ \\
Actuarial Graft Survival & & & \\
$\quad 1$ year & $90 \%$ & $88 \%$ & $89 \%$ \\
3 year & $84 \%$ & $76 \%$ & $80 \%$ \\
${ }^{*} \quad .031$ & & & \\
S. Creatinine (mg/dL) & $1.9 \pm 1.0$ & $1.9 \pm 1.8$ & $1.9 \pm 1.5$ \\
Off Steroids & $70 \%$ & $68 \%$ & $69 \%$ \\
Off Anti-Hypertensive & $39 \%$ & $36 \%$ & $38 \%$ \\
$\quad$ Medications & & & \\
\hline
\end{tabular}

Table 2. Tacrolimus in Renal Transplantation Pediatrics $n=43$

\begin{tabular}{lcc}
\hline Actuarial Survival & & \\
& 1 year & 3 year \\
& $100 \%$ & $100 \%$ \\
Patient Graft & $98 \%$ & $85 \%$ \\
S. Creatinine & $1.2 \pm 0.6 \mathrm{mg} / \mathrm{dL}$ & \\
Off Steroids & $62 \%$ & \\
Off Anti-Hypertensive & $62 \%$ & \\
$\quad$ Medications & & \\
\hline
\end{tabular}

ence between the two groups. The projected half-life of first cadaver kidneys was $11.9 \pm 2.5$ years.

Sixty-nine percent of successfully transplanted patients have been taken off steroids, and $38 \%$ are off antihypertensive medications. The mean serum creatinine is $1.9 \pm 1.5$ $\mathrm{mg} / \mathrm{dL}$. There are no differences between the double and triple drug groups in these parameters. The incidence of rejection was $50 \%$, and steroid-resistant rejection was seen in $11 \%$ of cases. There was a slightly lower, but not statistically different, incidence of rejection in the azathioprine group ( $45 \%$ vs $55 \%$ overall, and $8 \%$ vs $14 \%$ steroid resistance). There was also a substantial crossover between the two groups, more from triple to double therapy than vice versa.

Subsequent to completion of the second randomized trial, a third randomized trial was begun in December, 1993, evaluating the role of one week of low-dose cyclophosphamide in tacrolimus-treated recipients. Analysis of this trial is currently in progress, and a fourth randomized trial, comparing tacrolimus/prednisone and tacrolimus/prednisone/mycophenolate mofetil, has just begun.

\section{Pediatrics}

Between December, 1989 and December, 1993, 43 pediatric patients received kidneys under tacrolimus-based immunosuppression (concomitant or previous liver recipients were excluded from this analysis). ${ }^{2}$ With a mean follow-up of $25 \pm 14$ months, overall one- and three-year actuarial patient survival was $100 \%$ (Table 2). Overall one- and three-year actuarial graft survival was $98 \%$ and $85 \%$. Sixtytwo percent of the successfully transplanted children have

From the Pittsburgh Transplantation Institute (R.S., V.S., J.M., W.I., J.F., T.S.), Urologic Surgery (M.J., C.V., H.G., T.H.), Renal Electrolyte (D.E., N.G., S.L.L.), Transplant Pathology (P.R., A.D.), Surgery (R.S.), and the University of Pittsburgh Medical Center, Pittsburgh, Pennsylvania.

(C) 1996 by Appleton \& Lange

$0041-1345 / 96 / \$ 3.00 /+0$ 
been weaned off steroids, and $62 \%$ are not taking antihypertensive medications. The mean serum creatinine is $1.2 \pm 0.6 \mathrm{mg} / \mathrm{dL}$. The incidence of rejection was $58 \%$. and the incidence of steroid resistant rejection was $7 \mathrm{r}$. An additional 26 children were transplanted between January. 1994 and July, 1995, with $100 \%$ patient and graft survival.

The overall experience with tacrolimus in our renal transplant patients has been gratifying. Better short-term graft survival, longer projected half-lives, and a remarkable ability to wean from steroids in a majority of patients have been the most notable observations. Based on these findings, we believe that tacrolimus is a superior immunosuppressive agent in renal transplant recipients and is, at present, the drug of choice. The prolongation of cadaver kidney half-life in patients treated with tacrolimus is similar to that reported by Gjertson, Cecka, and Terasaki in a collection of cases compiled in the United Network for Organ Sharing (UNOS) Scientific Registry from 24 American renal transplant centers. ${ }^{9}$ Ongoing work is continuing to evaluate the role of new third agents and other modalities (eg bone marrow augmentation) $)^{10.11}$ in an effort to improve further the outcomes after transplantation.

\section{REFERENCES}

1. Starzl TE. Fung JJ. Jordan M. et al: JAMA 264:63, 1990)

2. Shapiro R. Scantlebury VP. Jordan ML, et al: Pediatric Nephrology 9:S43, 1995

3. Shapiro R. Jordan M. Fung J, et al: Trans Proc 23:920, 1991

4. Shapiro R. Jordan ML. Scantlebury V. et al: Trans Proc $23: 3065,1991$

5. Shapiro R. Jordan M. Scantlebury V, et al: Trans Proc 25:669, 1993

6. Shapiro R. Jordan M, Scantlebury V, et al: Clin Trans 8:508. 1994

7. Shapiro R, Jordan ML, Scantlebury VP, et al: Transplantation 59:485, 1995

8. Shapiro R, Jordan ML. Scantlebury VP, et al: Trans Proc 27(1):814, 1995

9. Gjertson DW, Cecka JM, Terasaki PI: Transplantation 60: 1384, 1995

10. Shapiro R, Rao AS, Fontes P, et al: Transplantation 59(2): 306, 1995

11. Shapiro R, Rao AS, Fontes P, et al: Transplantation 60:1421. 